\title{
Heat flux reconstruction in the grinding process from temperature data
}

\author{
J. Irša \& A. N. Galybin \\ Wessex Institute of Technology, Southampton, UK
}

\begin{abstract}
The problem considered in this paper deals with reconstruction of heat flux from temperature measurements, where due to lack of data, numerical integration is impossible. The problem is reduced to the determination of unknown complex coefficients of a linear piecewise holomorphic function representing heat flux properties and a quadratic holomorphic function representing complex temperature distribution. This leads to an over-determined system of linear algebraic equations, which are subjected to experimental errors. The reconstruction of heat flux is unique; however, reconstruction of flux directions is non-unique. It contains one free additive parameter. The method is useful in situations where limited data on temperature are provided.
\end{abstract}

Keywords: heat flux, temperature measurement, holomorphic function, complex variables.

\section{Introduction}

In heat flow problems, specifically in heat conductivity where a solid has varying temperature, information on the heat flux is essential. The measurement of heat flux is not an easy task and requires special devices, such as heat flux sensors. These sensors have a higher price than commonly used thermometers and the accuracy is also lower, as they are usually measure the difference in temperature.

In problems such as grinding, the heat flux is important due to thermal damage and residual stresses. In order to avoid that damage, many analytical solutions and numerical simulations were provided with different approaches to obtaining the heat flux shape $[1,2]$. The current assumption of heat flux entering the workpiece is triangular [2] or polynomial $\left(\mathrm{Q}=\mathrm{a}+\mathrm{bx}+\mathrm{cx}^{2}\right)[1]$. 
Heat flux is related to the gradient of temperature, therefore by having the temperature as a function, it is possible to obtain the heat flux very easily. However, the temperature is usually given in a dataset, only some locations or as an image.

Applying the complex variables theory to heat flow provides that the temperature is a harmonic function for steady state heat conductivity. Therefore, one can introduce the holomorphic function, which is a function of temperature and its complex harmonic conjugate. The derivative of the holomorphic function is related to the heat flux, which gives very useful properties.

The main idea is to approximate the holomorphic function of complex temperature distribution by the quadratic holomorphic function and complex heat flux by the linear holomorphic function in finite domains. These functions obey continuity along subdomain interfaces, on top of which two more equations are provided to utilize the data. A somewhat similar method was used in [3, 4] with stress trajectories.

The next section describes the theoretical background of steady state heat conduction, followed by problem formulation. The numerical method is explained with a discussion of uniqueness. The forthcoming sections are model examples, where ideal data are used to estimate accuracy as well as real data from the grinding process.

\section{Steady state temperature distribution}

When steady state prevails, the temperature function inside a $2 \mathrm{D}$ body $T(x, y)$ is harmonic $[5,6]$. Heat flux is mathematically defined by Fourier's Law:

$$
Q=-\operatorname{kgrad} T
$$

where $T(x, y)$ is temperature at point $(x, y)$ and $k$ refers to thermal conductivity of the material of the solid body.

The physical argument dictates that steady state temperature distribution prevails if there is no heat source or sink, which means there is no heat accumulation $\partial Q / \partial z=0$. Taking heat flux by its real and imaginary parts, representing $x$ and $y$ direction $Q=Q_{x}+i Q_{y}$, where

$$
Q_{x}=-k \frac{\partial T}{\partial x}, Q_{y}=-k \frac{\partial T}{\partial y}
$$

one will obtain the relation:

$$
\frac{\partial Q}{\partial z}=\frac{1}{2}\left(\frac{\partial Q_{x}}{\partial x}+\frac{\partial Q_{y}}{\partial y}\right)=0=-k \frac{1}{2}\left(\frac{\partial^{2} T}{\partial x^{2}}+\frac{\partial^{2} T}{\partial y^{2}}\right)
$$

The last expression (3) provides that the steady state temperature function $T$ is harmonic function. This provides existence of harmonic conjugate function $H$, where both of them satisfy CR; therefore we can write: 


$$
f(z)=T(x, y)+i H(x, y)
$$

where $f(z)$ is a holomorphic function called complex temperature distribution (CTD), where the real part of the function is associated with temperature. Very useful is the derivative of the CTD, which is related to the heat flux in somehow different form. Using the transformations in calculus and CR for the real and imaginary part of function $f(z) \partial T / \partial x=\partial H / \partial y, \partial H / \partial x=-\partial T / \partial y$ and substituting by (2) one will obtain the relationship between the derivative of CTD and heat flux, which will be referred to as a complex heat flux $(\mathrm{CHF})$ :

$$
\frac{\partial f}{\partial z}=\frac{1}{k}\left(-Q_{x}+i Q_{y}\right)=\frac{1}{k}|Q| e^{i \alpha}, \alpha=\pi-\varsigma
$$

where $|Q|$ is the value of the heat flux at a point and $\varsigma$ is the direction of the heat flux.

\section{Formulation of the problem}

Let $\Omega$ be a simply connected domain or a subdomain of a bigger domain which is not necessarily simply connected and bounded. Let temperature measurements be known at some discrete points $(j=1, \ldots, N)$, located within $\Omega$. The general problem is formulated as follows: given the discrete data on temperature $T_{j}=1 \ldots N$ find field of heat flux and heat flux directions within the domain $\Omega$.

No restrictions are imposed on data type. The data can be redistributed uniformly or randomly. To obtain the data from an image, a computer code has been designed by using MATLAB, which enables one to pick up the data from the screen from an image presenting temperatures.

\subsection{Uniqueness of reconstruction}

The problem is dealing with reconstruction of function that can be represented in complex exponential form $f(z)=\rho e^{i \omega}$, which leads to/from Cauchy-Riemann equations (CR). Therefore having one function known, the second one can be easily reconstructed by integration of CR. Indeed, assume, for instance, that the argument $\omega$ is known. Then by taking logarithm of both parts of, one obtains the following expression for holomorphic logarithmic function $\ln (f(z))=\ln (\rho(x, y))+i \omega(x, y)$. Therefore the logarithm of both right hand side parts must satisfy $\mathrm{CR}$, which allows one to find the former by integration:

$$
\ln (\rho(x, y))=-\int \frac{\partial \omega(x, y)}{\partial y} d x+p
$$

This formula indicates that the reconstruction can be achieved but it will always have one undetermined positive parameter $e^{p}$ as a free parameter. 
In the case when data are known at discrete points, reconstruction, in general, is non-unique and can have any finite number of free parameters, which is evident from the following. Let an exact solution $f(z)=u(x, y)+i v(x, y)$ of the problem be found by any method. It obviously, that the modulus must be real; therefore it satisfies the following:

$$
\operatorname{Im}\left\lfloor f\left(z_{j}\right) e^{-i \omega\left(z_{j}\right)}\right\rfloor=0, j=1 \ldots N
$$

Let us consider the polynomial, the roots of which are placed at the data points. This polynomial can be written in the form:

$$
P(z)=\sum_{j=1}^{m} C_{j}\left(z-z_{j}\right)^{m_{j}}
$$

where $C_{j}$ are arbitrary complex parameters and $m_{j}$ are arbitrary positive integers. It is evident from (8) that the sum, $f(z)+P(z)$, satisfies the condition (7) and therefore it is also the solution of the problem. Since $m_{j}$ are arbitrary, this solution has finite number of parameters defined as the degree of polynomial $P(z)$ plus one, and it is always greater than $N$.

In order to have sense the solution has to be sought in certain class of functions. Hereafter the solution is sought as a piecewise linear holomorphic functions. It will be shown that this set of functions allows reconstructing the flow with high accuracy.

Having the temperature function $T(x, y)$ known analytically $T(x, y)=a+b x+c y$ based on CR, one can reconstruct the CTD. The CTD will have therefore one free parameter $d$, affecting only $H$.

$$
f(z)=T(x, y)+i H(x, y)=a+b x+c y+i(-b y+d)
$$

After performing the derivative of (9), the free parameter $d$ will vanish. From this is obvious that the $\mathrm{CHF}$ and forthcoming heat flux can be reconstructed from temperature uniquely, however the complex conjugate function $H$ has free additive parameter.

\section{Method of solution}

The proposed method deals with reconstruction of complex temperature distribution (CDT) $f(z)$ from known discrete data on temperature. The domain is divided into $n$ smaller subdomains (elements) or arbitrary shape. In order to achieve piecewise linear function of $\mathrm{CHF}$, the CTD must be approximated by quadratic function. Both these approximations obey continuity along all interfaces (at certain collocation points located on the interfaces). For complex heat flux is required approximating function piece-wise linear function:

$$
\chi_{C H F}^{(m)}(z)=a^{(m)}+b^{(m)} z, \quad m=1 \ldots n
$$


for complex temperature distribution is therefore the approximating function the integration of that function:

$$
\chi_{C T D}^{(m)}(z)=c^{(m)}+a^{(m)} z+\frac{1}{2} b^{(m)} z^{2}, \quad m=1 \ldots n
$$

Here $\quad a^{(m)}=a_{0}^{(m)}+i a_{1}^{(m)}, b^{(m)}=b_{0}^{(m)}+i b_{1}^{(m)}, c^{(m)}=c_{0}^{(m)}+i c_{1}^{(m)} \quad$ are $\quad 3$ unknown complex constants; therefore 6 real constants are unknown in every element. Different approximating function could be used instead of linear holomorphic function for complex heat flux. It will be demonstrated that the use of these two particular functions provides results with very high accuracy.

\subsection{Discretization of the domain}

The grid used in this thesis consists of square elements with collocation points on its interfaces. The latter are necessary in order to obey continuity between the elements. The collocation points (CP) are distributed regularly. An example, of such a grid with known data is shown on (Figure 1).

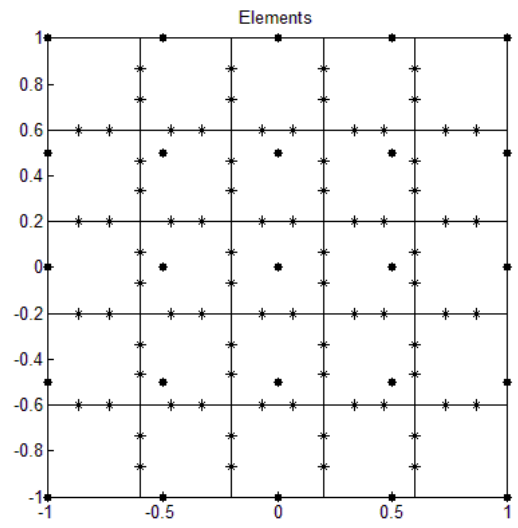

Figure 1: Discretization of the domain by square elements, collocation points - stars, data - dots.

The minimum number of nodes strictly depends on the number of unknowns in the approximating function as well as the element type. Given that, the square elements are placed in the regular grid with $J$ elements along $x$-axis and $K$ elements along $y$-axis and 6 unknown coefficients in every element, one finds the total number of unknowns $N_{u n k n}$ as:

$$
N_{\text {unkn }}=6 N_{\text {elem }}=6 J K
$$

The required number of collocation points depends on the number of element interfaces, which is $N_{\text {int }}=(J-1) K+(K-1) J$. Using the same number of collocation points per each interface, keeping in mind that separation of complex 
equations into real and imaginary parts presents two real equations at every collocation point, the required number of collocation points per interface is:

$$
N_{c p} \geq \frac{N_{\text {unkn }}}{2_{\text {int }}}=\frac{6 J K}{2[(J-1) K+(K-1) J]}=\frac{3 J K}{2 J K-J-K}
$$

For the domain, where $J$ or $K$ is equal to 1 , the number of collocation points per interface is $N_{c p} \geq 3$. In general case the number of collocation points per interface $N_{c p}>2$. Due to condition equations imposed, the $N_{c p}$ will be lowered; therefore $N_{c p}=2$ is used further on.

There are no formal restrictions in the mesh used. Other types of mesh could be used, such as triangular or polygonal with the straight or curvilinear sides. The square grid is convenient from computational point of view because the distances between collocation points are regular, which has good influence on the structure of the matrix of the system of linear equations that is expressed in lower condition numbers of the matrix of the system.

\subsection{Equations of continuity and condition equations}

This problem consists of two continuity equations and two types of condition equations. For the $k$-th collocation point, lying on the interface between the elements numbered $m$ and $m+1$, the equation of continuity for CTD is following:

$$
\chi_{C T D}^{(m)}\left(z_{k}\right)-\chi_{C T D}^{(m+l)}\left(z_{k}\right)=0, \quad m=1 \ldots n ; \quad k=1 \ldots N_{C P}
$$

The second equation of continuity for $\mathrm{CHF}$ is expressed following:

$$
\chi_{C H F}^{(m)}\left(z_{k}\right)-\chi_{C H F}^{(m+l)}\left(z_{k}\right)=0, \quad m=1 \ldots n ; \quad k=1 \ldots N_{C P}
$$

These equations present the first set of equations for the system of linear algebraic equations (SLAE). The condition equation containing $T$ comes from CTD, where the real part of it is equal to the temperature, therefore:

$$
\operatorname{Re}\left(\chi_{C T D}^{(m)}\left(z_{j}\right)\right)=T_{j}, \quad j=1 \ldots N
$$

this condition equation is satisfied in elements, where the data occurs. The second condition equation is due to free parameter $c_{1}$. As was mentioned, we are unable from temperature data obtain the parameter $c_{1}$, therefore from equations (14), (15) and (16) we are able to recover only the differences of those constants between adjacent elements. Therefore specific condition is used, which provides the sum of all constants $c_{1}$ across whole domain zero. The complex conjugate function $H$, can be obtained by adding any real parameter. The condition is following:

$$
\sum_{i=1}^{N} \operatorname{Im}\left(c^{(i)}\right)=0
$$


The SLAE formed by equations (14)-(17) is over-determined, it has $4 N_{\text {int }} N_{c p}+N$ equations and $6 N_{\text {elem }}$ unknowns.

Should be noticed that the system can be solved even without the equation (17), however this would decrease the rank of matrix by one, and therefore higher $\mathrm{CN}$ would arise and then regularization has to be used.

\subsection{Solution of the linear system}

By extracting the real and imaginary parts of complex equations (equations of continuity and condition equations) the real SLAE can be obtained and rewritten in a matrix form:

$$
A x=b
$$

where $A \in R^{m x n}, b \in R^{m}$ and $m>n . x$ is the vector of the unknown real coefficients, of length $n$. The vector $x$ consists of real and imaginary parts of unknown complex coefficients. Vector $b$ is known exactly from temperature measurements, while $A$ the matrix of the SLAE depends on approximating functions, type and size of element. The matrix $A$ is not a square, the system is over-determined and therefore the left-hand side $A x$ does not exactly equal to $b$ and thus the system is inconsistent. However an approximate solution, $\mathrm{x}^{*}$, can be found by means of the least squares methods [5] (LSQR) that minimise the residual:

$$
\|A x *-b\|_{2} \leq \varepsilon
$$

where $\|. .\|_{2}$ stands for the $L_{2}$ norm. If the arising system is well-posed and not large, then the inversion of the matrix does not meet any difficulties and solution takes the form [7]:

$$
x^{*}=\left(A^{T} A\right)^{-1} A^{T} b
$$

The condition number $(C N)$ is used in the numerical examples to control wellposedness of the SLAE. In the case of high CN, the methods for ill-posed problems should used [8].

\section{Model examples}

\subsection{Synthetic example: $f(z)=1+z+z^{2}+z^{3}$}

The CTD function is chosen as $f(z)=1+z+z^{2}+z^{3}$. The thermal conductivity is chosen as $k=1.64$ uniformly distributed data were chosen and 169 elements. This provides 2561 equations, consisting of 1248 equations of continuity for $\mathrm{CTD}$ and the same number for CHF, 64 condition equations from known data and 1 condition equation due to free parameter. The system consisted of 1014 unknown parameters. $\mathrm{CN}$ of that system was 371 with errors in the heat flux: maximum error $4.3 \%$ and average error $1.3 \%$. The residual was 15.9 . Comparisons between ideal and reconstructed temperature, heat flux and temperature profiles are shown in (Figure 2, Figure 3, and Figure 4). 


\subsection{Experimental data 2 - real data (grinding)}

Real data on temperature were obtained from an image of work piece temperature after treatment, coming from grinding process (Figure 5). The data were picked from [1]. In this example are two types of errors. The first is due to numerical integration of Planck's law used in [1] and second is due to manual picking up data (section: data description). There was 105 picked data and 400 elements used for the reconstruction. The arising system consisted of 6187 equations and 2400 unknowns. The condition number was $\mathrm{CN}=2.8 \mathrm{e} 3$ and residual $6.3 \mathrm{e} 3$.

The reconstructed temperature and heat flux is shown in (Figure 6).

Heat flux coming to the workpiece on its surface is shown in (Figure 7, left). The heat flux directions are shown in (Figure 7, right); however it was shown that one parameter remains free. This means that, to obtain the real directions, one constant has to be added to the reconstructed directions.

\section{Conclusion}

The present study investigates the heat flux shape, based on temperature measurements. The method comes from complex variable theory applied to
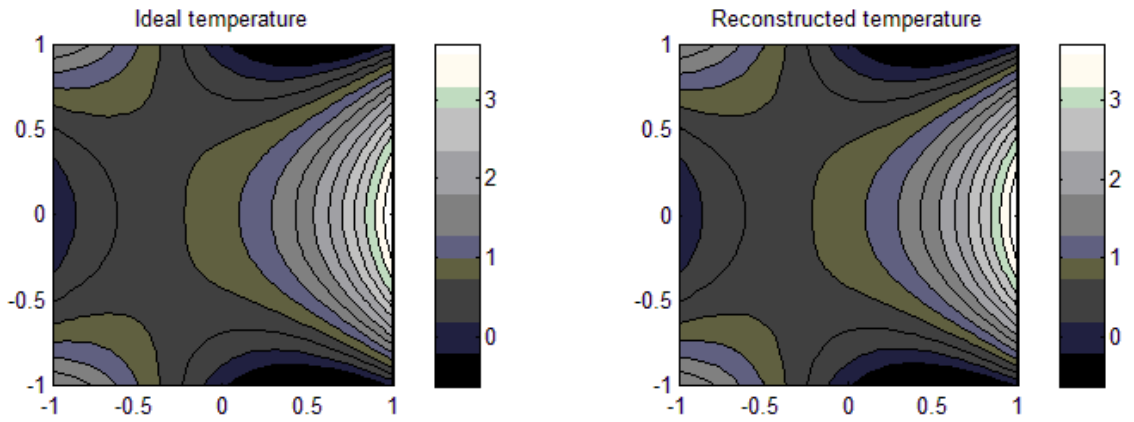

Figure 2: $\quad$ Temperature comparisons, ideal - left and reconstructed - right.
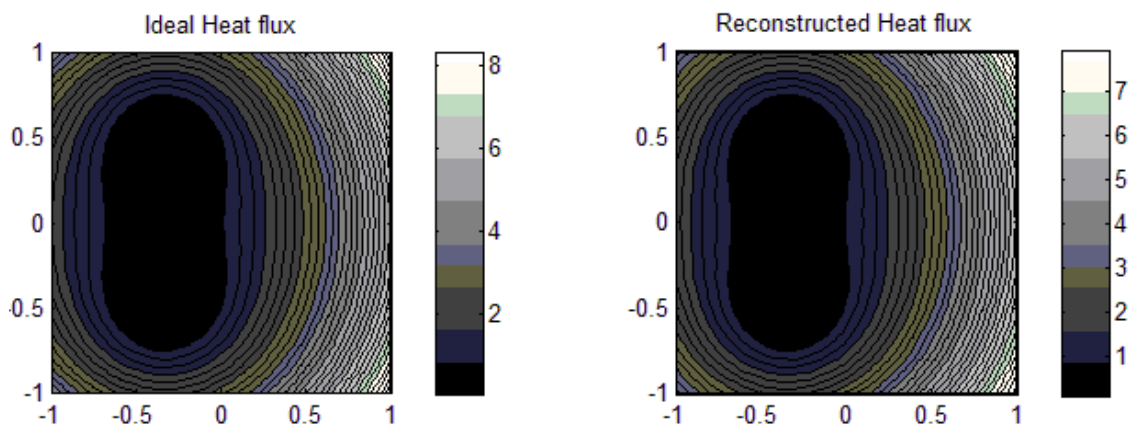

Figure 3: Heat flux comparison, ideal - left and reconstructed - right. 

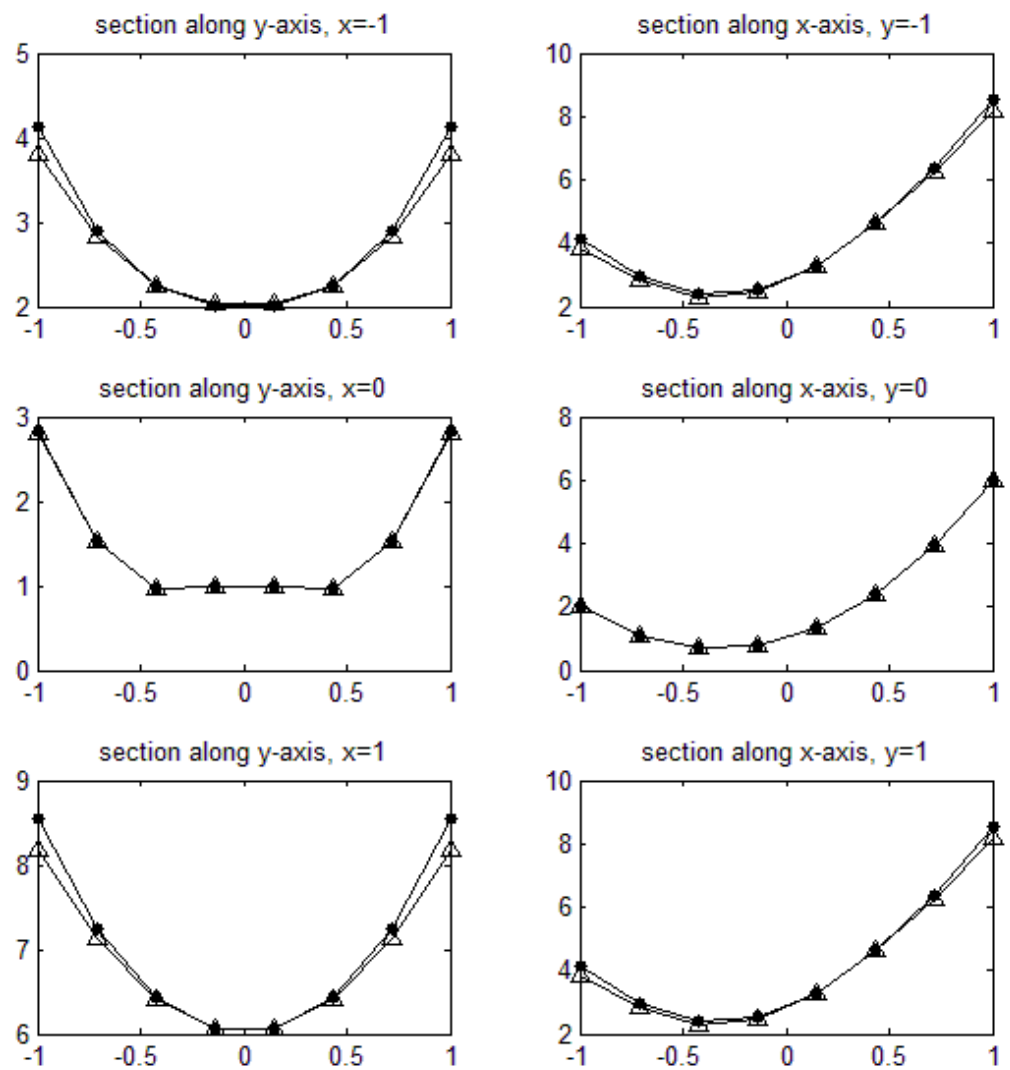

Figure 4: $\quad$ Profiles of heat flux, ideal - dots and reconstructed - triangles.

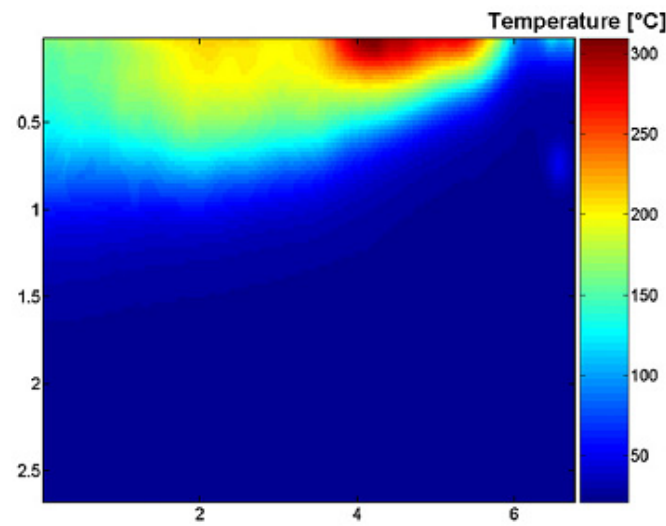

Figure 5: Data on temperature of the workpiece after treatment derived from experiment [1]. 

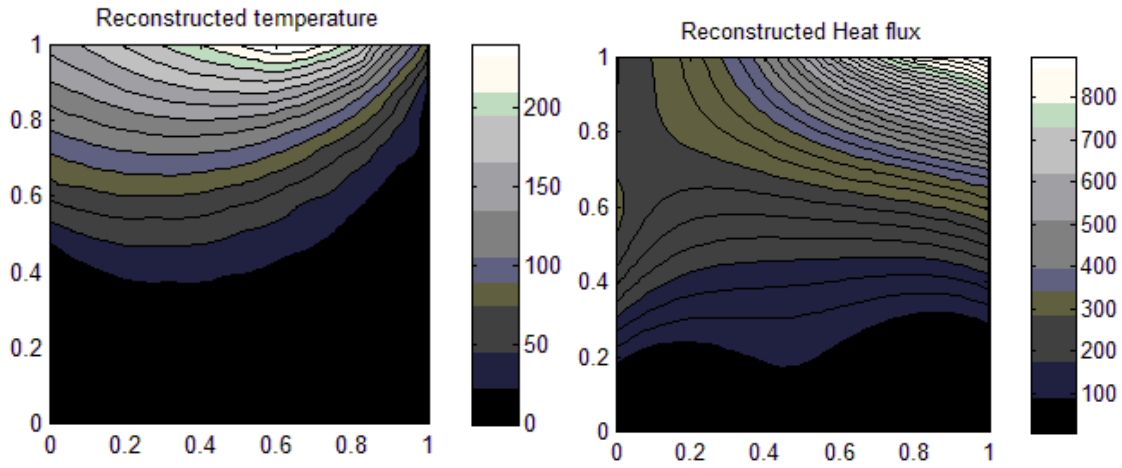

Figure 6: Reconstructed temperature of the workpiece - left and recovered heat flux - right.
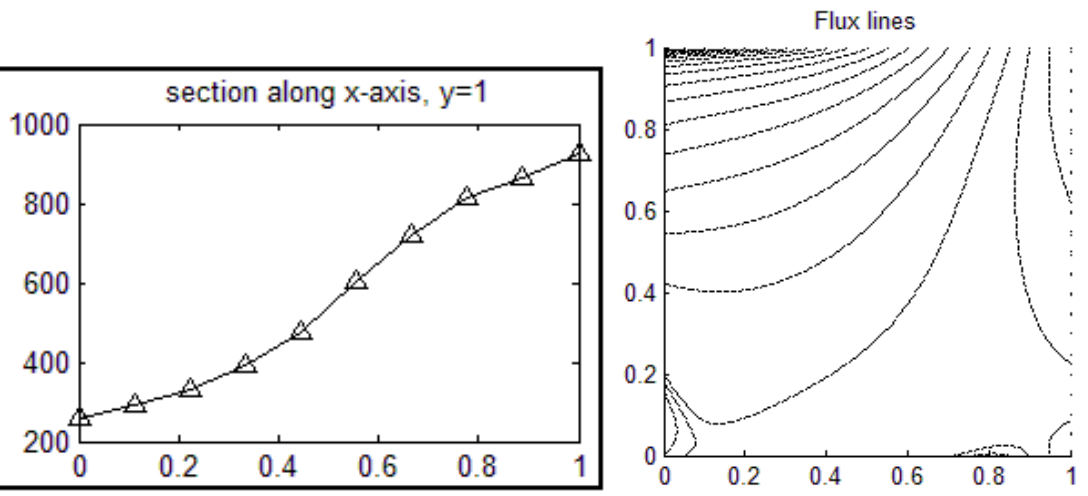

Figure 7: Recovered heat flux profile on the grinded surface - left and heat flux direction - right.

steady state heat flow, where holomorphic function arises. The real part of the function is related to temperature and the derivative is related to the heat flux. This theory was used to reconstruct the heat flux from temperature data, when the numerical integration, cannot be performed due to lack of data. The method was tested on ideal data where the errors were below $2 \%$. In case of real measurement data, an image of workpiece after treatment resulting from thermography [1] was used. It was shown in the experimental data, that even with very high errors the method is able to provide reasonable results. Moreover it was shown that the common triangular shape of heat flux [2] or polynomial of second order [1] are not accurate enough to represent the shape of entering heat flux at the surface of workpiece during the grinding process based on the data which were used. This method has recovered also the flux directions with one free additive parameter. 


\section{Acknowledgement}

The authors are grateful to EPSRC for the financial support of this work through the Research Grant EP/E032494/1.

\section{References}

[1] A. Brosse, P. Naisson, H. Hamdi, J.M. Bergheau, Temperature measurement and heat flux characterization in grinding using thermography, JMATPROTEC, 201, 2008, 590-595.

[2] S. Malkin, C. Guo, Model Based Simulation of Grinding Process.

[3] A.N. Galybin and Sh.A. Mukhamediev, Determination of elastic stresses from discrete data on stress orientations, IJSS, 41 (18-19), 2004, 5125 5142.

[4] Sh.A. Mukhamediev, A.N. Galybin and B.H.G. Brady, Determination of stress fields in elastic lithosphere by methods based on stress orientations, Int J Rock Mech Min,43 (1), 2006, 66-88.

[5] M. Rahman, Complex Variables and Transform Calculus, Computational Mechanics Publications, Southampton, 1997.

[6] Y.K. Kwok, Applied complex variables, Cambridge University Press, Cambridge, 2002.

[7] G.H. Golub, C.F. Van Loan, Matrix Computations, London: The John Hopkins Press Ltd., 1996.

[8] A.N. Tikhonov, V.Y. Arsenin, Solution of Ill-posed Problems, Winston, Wiley, New York, 1977. 